\title{
Knockdown of heme oxygenase-1 promotes apoptosis and autophagy and enhances the cytotoxicity of doxorubicin in breast cancer cells
}

\author{
XIAO-FENG ZHU ${ }^{1 *}$, WEN LI $^{2,3^{*}}$, JIE-YI MA ${ }^{2}$, NAN SHAO ${ }^{1}$, YUN-JIAN ZHANG ${ }^{1}$, RUI-MING LIU ${ }^{2,3}$, \\ WEI-BIN WU ${ }^{1}$, YING LIN $^{1}$ and SHEN-MING WANG ${ }^{1,3}$ \\ ${ }^{1}$ Department of Breast and Thyroid Surgery, ${ }^{2}$ Laboratory of General Surgery, \\ ${ }^{3}$ Guangdong Engineering Laboratory for Diagnosis and Treatment of Vascular Disease, \\ The First Affiliated Hospital of Sun Yat-Sen University, Guangzhou, Guangdong 510080, P.R. China
}

Received November 5, 2014; Accepted May 29, 2015

DOI: 10.3892/ol.2015.3735

\begin{abstract}
Heme oxygenase-1 (HMOX-1) is a microsomal enzyme that exerts anti-apoptotic and cytoprotective effects. In the present study, HMOX-1 was demonstrated to be overexpressed and able to be induced by doxorubicin in breast cancer cell lines. Knockdown of $H M O X-1$ using short interfering (si)RNA enhanced the cytotoxicity of doxorubicin in MDA-MB-231 and BT549 cells. Knockdown of HMOX-1 downregulated $\mathrm{B}$ cell lymphoma $(\mathrm{Bcl})-2$ and Bcl-extra large expression, and significantly enhanced doxorubicin-induced apoptosis in MDA-MB-231 and BT549 cells. Additionally, knockdown of HMOX-1 upregulated light chain 3B expression and markedly increased the accumulation of autophagic vacuoles in MDA-MB-231 and BT549 cells treated with doxorubicin. These results indicated that HMOX-1 may be involved in conferring the chemoresistance of breast cancer cells, by preventing apoptosis and autophagy. Therefore, HMOX-1 may represent a potential therapeutic target for enhancing the cytotoxicity and efficacy of doxorubicin during the treatment of breast cancer.
\end{abstract}

\section{Introduction}

Breast cancer is the most frequently diagnosed cancer amongst females and the second most common cause of

Correspondence to: Professor Shen-Ming Wang or Professor Ying Lin, Department of Breast and Thyroid Surgery, The First Affiliated Hospital of Sun Yat-Sen University, 58 Zhongshan $2^{\text {nd }}$ Road, Guangzhou, Guangdong 510080, P.R. China

E-mail: shenmingwang@hotmail.com

E-mail: frostlin@hotmail.com

*Contributed equally

Key words: heme oxygenase-1, apoptosis, autophagy, doxorubicin, breast cancer, chemoresistance cancer-related mortality among women (1). One third of novel cancer diagnoses in females are breast cancer and a total of one in eight women will be diagnosed with breast cancer, with a lifetime risk of mortality due to breast cancer of $3.4 \%$ (1). Chemotherapy is one of the major systemic therapies available for the treatment of breast cancer. A recent meta-analysis of the outcome of 100,000 patients with breast cancer, confirmed the benefit of cyclophosphamide-, methotrexate- and fluorouracil- therapies and anthracycline-based therapies with absolute reduction of mortalities of 6.2 and $6.5 \%$, respectively, at 10 years (2). Despite progress in the improvement of chemotherapeutic strategies, the ability to treat advanced breast cancer remains poor, mainly due to the chemoresistance of cancer cells to standard chemotherapy. Numerous anti-apoptotic and cytoprotective pathways have been associated with the chemoresistance of cancer cells $(3,4)$.

Heme oxygenase (HMOX) is a microsomal enzyme, which catalyzes the initial, rate-limiting step in the degradation of heme, and has a crucial role in the recycling of iron (5). The enzymatic activity of HMOX also produces $\mathrm{CO}$, ferrous iron and biliverdin. Thus, HMOX is able to reduce oxidative stress, attenuate inflammatory responses and lower the rate of apoptosis (6). HMOX-1, an isoform of HMOX, may be induced in response to cellular stress and diverse oxidative stimuli (7). HMOX-1 is frequently overexpressed in a range of cancers, including hepatoma, prostate cancer, melanoma and brain tumors (8-11). HMOX-1 promotes proliferation in human melanoma and pancreatic cancer cell lines $(10,12)$. As HMOX-1 is a potent inducer of vascular endothelial growth factor (VEGF), a crucial factor involved in tumor angiogenesis, it has also been recognized to stimulate angiogenesis and thus support tumor progression (13). Additionally, overexpression of HMOX-1 promoted metastasis in a murine model of melanoma (14).

Expression of HMOX-1 may be induced by radiation and chemotherapy in cancer cells (15), and the expression of HMOX-1 was predictive of poorer survival in patients with breast carcinoma (16). HMOX-1 has also been found to protect cancer cells against apoptosis by exerting 
anti-apoptotic activities $(17,18)$. Studies have indicated that upregulation of HMOX-1 is correlated with the inhibition of autophagy $(19,20)$. Autophagy, which describes the process of cellular self-digestion, is considered to be a cytoprotective response, which may occur following the withdrawal of growth factors or under stressful conditions (21). However, under certain conditions, autophagy may function as a mechanism of cell death (22). HMOX-1 and autophagy are induced in response to cell stress $(15,21)$. Therefore, HMOX-1 may function as a specific regulator of autophagy in breast cancer and may have a role in the survival of breast cancer cells during chemotherapy.

In the present study, HMOX-1 expression and the effects of doxorubicin in breast cancer cell lines were evaluated. In addition, the effects of HMOX-1 knockdown in breast cancer cells were investigated.

\section{Materials and methods}

Cell culture. The human breast cancer cell lines MDA-MB-231, BT549, HS578T, MDA-MB-468, MCF-7 and ZR751, as well as the non-malignant MCF-10A breast cell line were purchased from the American Type Culture Collection (Manassas, VA, USA) and cultured in Dulbecco's modified Eagle's medium (DMEM) supplemented with $10 \%$ fetal bovine serum and $1 \%$ penicillin and streptomycin (Gibco, Karlsruhe, Germany) in a $5 \% \mathrm{CO}_{2}$ atmosphere at $37^{\circ} \mathrm{C}$. Doxorubicin was obtained from Keygen Biotech (Nanjing, China), dissolved in water and stored at $4^{\circ} \mathrm{C}$.

Transfection of short interfering (si)RNAs. HMOX-1 siRNA and control siRNA was synthesized by Guangzhou RiboBio Co., Ltd (Guangzhou, China). The sequence of HMOX-1 siRNA was as follows: Sense, 5'-CCAGCAACAAAGUGC AAGAdTdT-3' and antisense, 3'-dTdTGGUCGUUGUUUCACGUUCU-5'. Breast cancer cell lines were transfected with $50 \mathrm{nM}$ siRNA using RNAiMAX transfection reagent (Invitrogen Life Technologies, Carlsbad, CA, USA) according to the manufacturer's instructions.

Reverse transcription quantitative-polymerase chain reaction (RT-qPCR) for mRNA quantification. Total RNA was extracted from the cells using TRIzol (Invitrogen Life Technologies) and complementary (c)DNA was synthesized from 1,000 ng total RNA using the PrimeScript RT reagent kit (Takara Biotechnology, Co., Ltd, Dalian, China), according to the manufacturer's instructions. PCR was performed using the PrimeScript RT Master Mix (Takara Biotechnology, Co., Ltd) on a Bio-Rad CFX96 real-time PCR machine (Bio-Rad Laboratories, Inc., Hercules, CA, USA). The primer sequences were as follows: $H M O X-1$ forward, 5'-TACCACATC TATGTGGCCCTG-3' and reverse, 5'-TGGCTGGTG TGTAGGGGAT-3'; GAPDH forward, 5'-GCACCGTCA AGGCTGAGAAC-3' and reverse, 5'-TGGTGAAGACGC CAGTGGA-3'. Data analysis was performed using the comparative $\mathrm{C}_{\mathrm{T}}$ method (23) with Bio-Rad Manager 2.1 software (Bio-Rad Laboratories, Inc.).

Western blotting. Cells were lysed in radioimmunoprecipitation assay lysis buffer (Cell Signaling Technology, Inc.
Danvers, MA, USA) supplemented with protease inhibitor (Roche Diagnostics, Basel, Switzerland). The concentration of total protein was determined using a bicinchoninic acid assay kit (Keygen Biotech). Equal amounts of protein (35 $\mu \mathrm{g})$ were separated by $10 \%$ SDS-PAGE, transferred onto polyvinylidene difluoride membranes (EMD Millipore, Bedford, MA, USA) and blotted with the following monoclonal IgG rabbit anti-human antibodies at a dilution of 1:1,000: Anti-HMOX-1 (cat. no. ab52947; Abcam, Cambridge, UK), anti-B cell lymphoma-2 (Bcl-2; cat. no. 2870), anti-Bcl-extra large (xL; cat. no. 2764), anti-Bcl-2-like protein 4 (Bax; cat. no. 5023), anti-Beclin-1 (cat. no. 3495), anti-LC3B (cat. no. 3868) or anti- $\beta$-tubulin (cat. no. 2128) (Cell Signaling Technology, Inc.), followed by incubation overnight at $4^{\circ} \mathrm{C}$. The bands were visualized using Luminol reagent (Pierce Biotechnology, Inc.; Thermo Fisher Scientific, Inc., Waltham, MA, USA) and imaged using GE ImageQuant Las 4000 mini (GE Healthcare Life Sciences, Chalfont, UK).

Drug sensitivity assay. MDA-MB-231 and BT549 cells were seeded in 96-well culture plates at a density of 5,000 and 10,000 cells/well, respectively, and transfected with siRNA, $12 \mathrm{~h}$ prior to treatment with $0.02,0.05,0.10,0.15$ or $0.20 \mu \mathrm{g} / \mathrm{ml}$ doxorubicin for $48 \mathrm{~h}$. Cell viability was assessed using the Cell Counting kit-8 (CCK-8; Dojindo, Kumamoto, Japan) according to the manufacturer's instructions. The absorbance values were measured at $450 \mathrm{~nm}$ using a Sunrise Absorbance Reader (Tecan US, Inc., Morrisville, NC, USA).

Cell apoptosis assay. Apoptosis was assessed using an Annexin V-fluorescein isothiocyanate (FITC) apoptosis kit (Dojindo) according to the manufacturer's instructions. Briefly, the cells were transfected with siRNA for $12 \mathrm{~h}$ prior to treatment with $0.2 \mu \mathrm{g} / \mathrm{ml}$ doxorubicin for $48 \mathrm{~h}$. The cells were harvested, resuspended in $500 \mu \mathrm{l}$ binding solution, and incubated with $5 \mu \mathrm{l}$ Annexin $\mathrm{V}$ and $5 \mu \mathrm{l}$ propidium iodide (PI) for $10 \mathrm{~min}$ in the dark. Apoptosis was analyzed by flow cytometry using a Beckman Coulter EPICS XL-MCL and the results were analyzed using Kaluza software version 1.2 (both from Beckman Coulter Inc., Brea, CA, USA).

Cell autophagy assay. MDA-MB-231 and BT549 cells were seeded in 24-well plates at a density of 20,000 and 40,000 cells/well, respectively, and transfected with siRNA for $12 \mathrm{~h}$ prior to treatment with $0.1 \mu \mathrm{g} / \mathrm{ml}$ doxorubicin for $48 \mathrm{~h}$. The cells were then washed in phosphate-buffered saline (PBS; Gibco Life Technologies, Shanghai, China), fixed in 4\% formaldehyde (Xilong Chemical Co., Ltd., Shantou, China) for $15 \mathrm{~min}$, permeabilized with 0.1\% Triton X-100 (Beyotime Institute of Biotechnology, Shanghai, China) for $10 \mathrm{~min}$, blocked with $0.1 \%$ bovine serum albumin (Beyotime Institute of Biotechnology) for $1 \mathrm{~h}$ and incubated with anti-light chain 3B (LC3B) antibody (Cell Signaling Technology, Inc.) overnight at $4^{\circ} \mathrm{C}$. Subsequently, cells were incubated with a green fluorescent protein (GFP)-tagged secondary antibody (Life Technologies, Grand Island, NY, USA) for $1 \mathrm{~h}$ at room temperature, then incubated with DAPI (Roche Diagnostics) for $10 \mathrm{~min}$ and images were acquired using a fluorescence microscope (Carl Zeiss Axio Observer Z1; Carl Zeiss, Jena, Germany). 
A

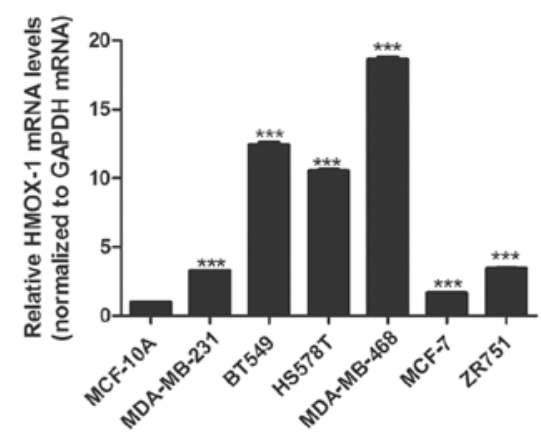

B

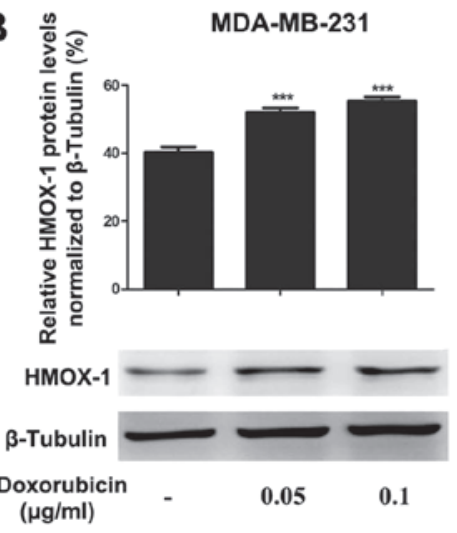

C

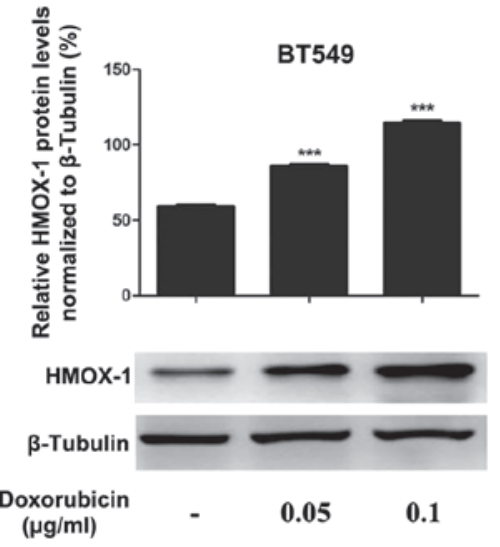

D

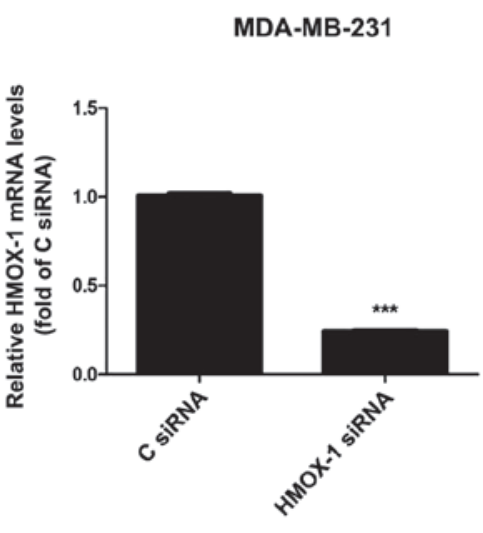

E

Figure 1. HMOX-1 is overexpressed and is induced by doxorubicin in breast cancer cell lines. (A) RT-qPCR analysis of $H M O X-1$ expression in breast cancer cell lines and MCF-10A non-malignant breast cells. (B and C) Western blot analysis of HMOX-1 protein expression in (B) MDA-MB-231 and (C) BT549 cells treated with various concentrations of doxorubicin for 48 h. (D and E) RT-qPCR analysis of HMOX-1 expression in (D) MDA-MB-231 and (E) BT549 cells $48 \mathrm{~h}$ following transfection with $50 \mathrm{nM} H M O X-1$ siRNA or control siRNA. All experiments were repeated at least three times. Data are presented as the mean \pm standard deviation. ${ }^{* * *} \mathrm{P}<0.001$ vs. control cells. HMOX-1, heme oxygenase-1; RT-PCR, reverse transcription-quantitative polymerase chain reaction; mRNA, messenger RNA; siRNA, short interfering RNA; C, control.

Statistical analysis. Data are expressed as the mean \pm standard deviation. Differences between the treatment groups and control group were assessed with Student's t-test using GraphPad Prism version 5.0 (GraphPad Software Inc., San Diego, CA, USA). $\mathrm{P}<0.05, \mathrm{P}<0.01$ and $\mathrm{P}<0.001$ were considered to indicate statistically significant differences.

\section{Results}

HMOX-1 is overexpressed and able to be induced by doxorubicin in breast cancer cell lines. RT-qPCR analysis indicated that $H M O X-1$ was overexpressed in the breast cancer cell lines evaluated. Compared with that of the non-malignant breast cell line MCF-10A, the expression of $H M O X-1$ was 3.3-fold higher in MDA-MB-231 cells, 12.4-fold higher in BT549 cells, 10.5-fold higher in HS578T cells, 18.6-fold higher in MDA-MB-468 cells, 1.6-fold higher in MCF-7 cells and 3.4-fold higher in ZR751 cells (Fig. 1A). In addition, doxorubicin treatment significantly upregulated $\mathrm{HMOX}-1$ protein expression in MDA-MB-231 and BT549 cells (Fig. 1B and C).

Silencing HMOX-1 increases the sensitivity of breast cancer cell lines to doxorubicin. A cytotoxicity assay was performed to investigate the effects of HMOX-1 on the sensitivity of breast cancer cell lines to doxorubicin. Breast cancer cells were transfected with $50 \mathrm{nM} H M O X-1$ siRNA or the negative control siRNA. At $48 \mathrm{~h}$ post-transfection, $H M O X-1$ messenger (m)RNA expression was significantly knocked down in the MDA-MB-231 and BT549 cell lines (Fig. 1D and E). The transfected cells were subsequently exposed to $0.02,0.05$, $0.1,0.15$ or $0.2 \mu \mathrm{g} / \mathrm{ml}$ doxorubicin for $48 \mathrm{~h}$. The CCK-8 assay demonstrated that knockdown of $H M O X-1$ significantly enhanced the cytotoxicity of doxorubicin in MDA-MB-231 and BT549 cells (Fig. 2).

HMOX-1 knockdown enhances doxorubicin-induced apoptosis in breast cancer cell lines. MDA-MB-231 and BT549 cells were transfected with $H M O X-1$ siRNA or control siRNA, treated with $0.2 \mu \mathrm{g} / \mathrm{ml}$ doxorubicin for $48 \mathrm{~h}$, then stained using Annexin V and PI and subjected to flow cytometric analysis to quantify apoptotic cells. Silencing of $H M O X-1$ significantly increased the apoptotic rate in doxorubicin-treated MDA-MB-231 and BT549 cells (Fig. 3A and B). Furthermore, western blot analysis indicated that knockdown of $H M O X-1$ reduced the expression of $\mathrm{Bcl}-\mathrm{xL}$ and $\mathrm{Bcl}-2$, but did not alter the expression of Bax (Fig. 3C).

HMOX-1 silencing enhances doxorubicin-induced autophagy in breast cancer cell lines. Doxorubicin is able to promote cell death in cancer cells via autophagy (21). Silencing 
A

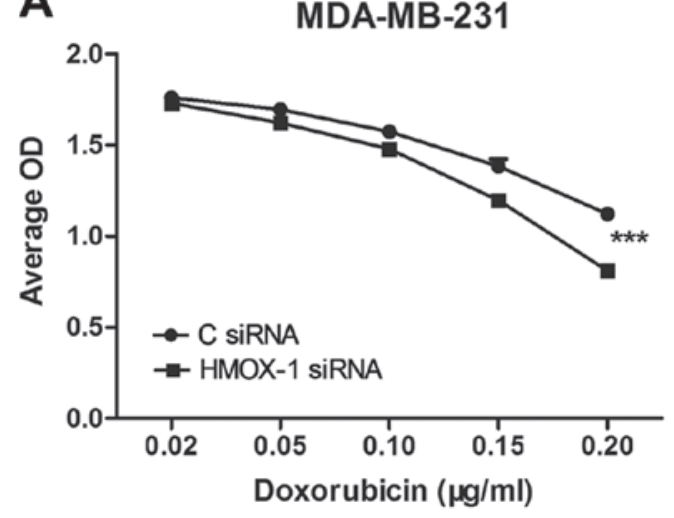

B

BT549

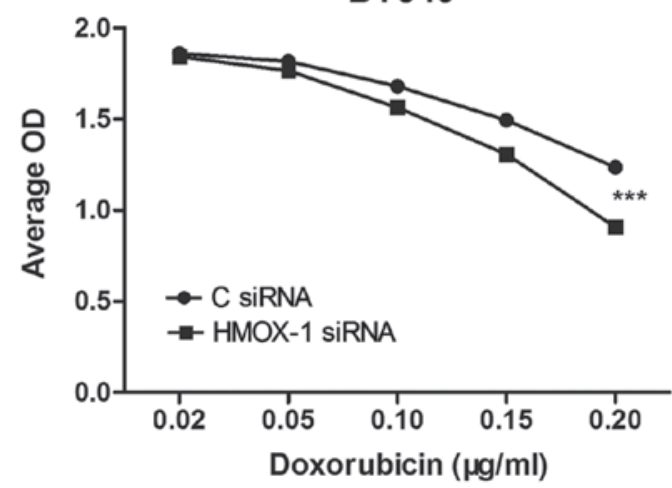

Figure 2. Knockdown of HMOX-1 increases the cytotoxicity of doxorubicin in breast cancer cell lines. (A) MDA-MA-231 and (B) BT549 cells were transfected with C siRNA or HMOX-1 siRNA and then exposed to various concentrations of doxorubicin for $48 \mathrm{~h}$. Cell viability was analyzed using the cell counting kit-8 assay. All experiments were repeated at least three times. Data are presented as the mean \pm standard deviation. ${ }^{* * *} \mathrm{P}<0.001$ vs. control cells. HMOX-1, heme oxygenase-1; C, control; siRNA, short interfering RNA; OD, optical density.

A
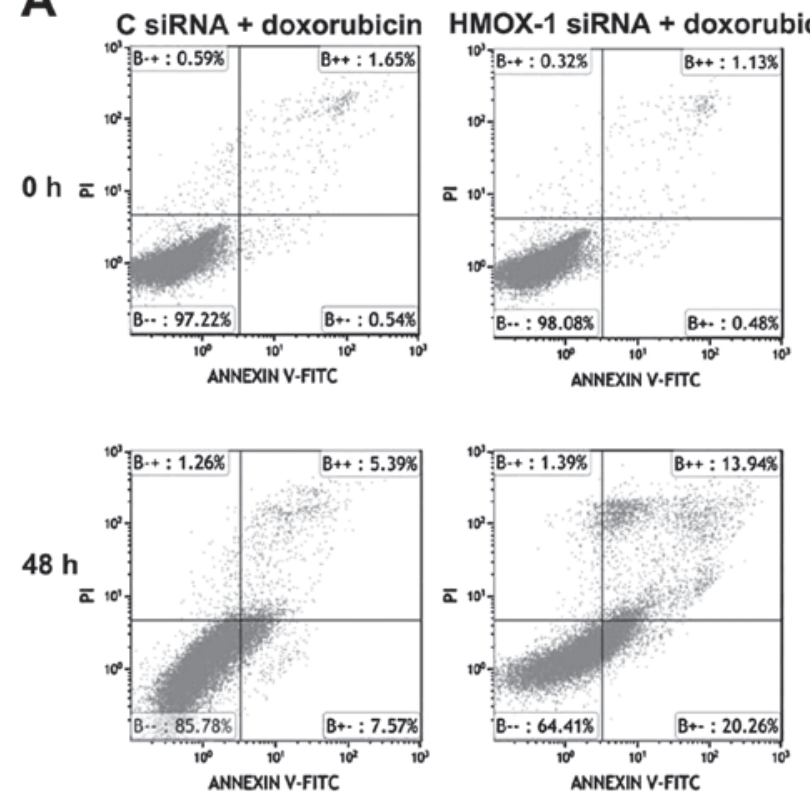

C
B
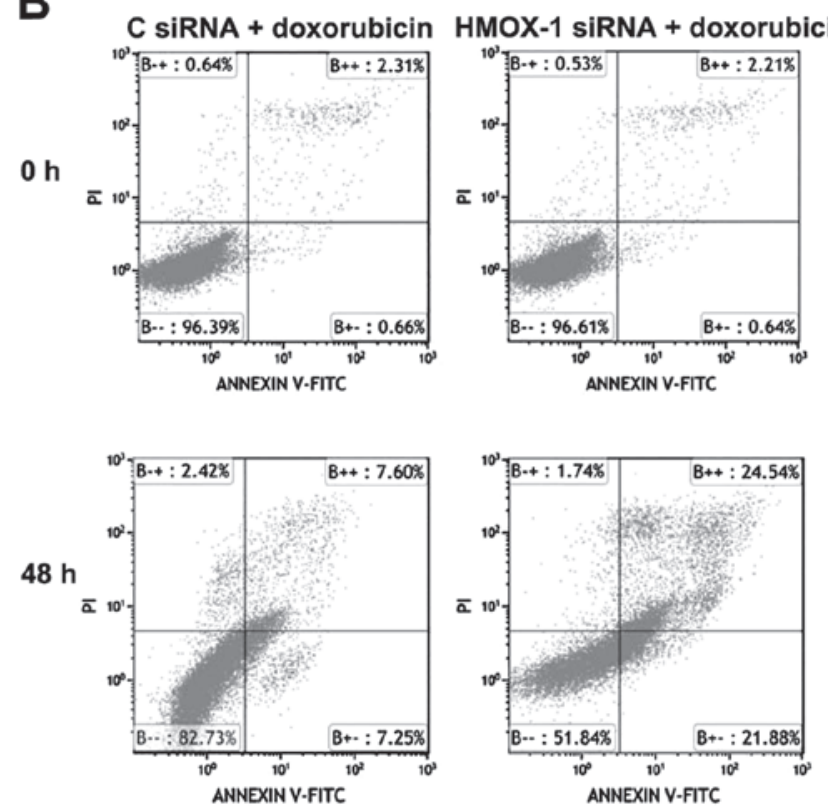

BT549

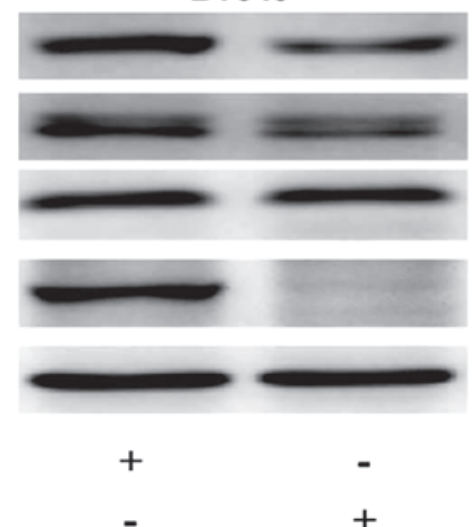

Figure 3. Knockdown of $H M O X-1$ enhances doxorubicin-induced apoptosis in breast cancer cell lines. (A and B) Rate of apoptosis in (A) MDA-MB-231 cells and (B) BT549 cells transfected with C siRNA or HMOX-1 siRNA and then treated with $0.20 \mu \mathrm{g} / \mathrm{ml}$ doxorubicin for $48 \mathrm{~h}$. The rates of apoptosis were determined using Annexin V and PI staining and flow cytometry. (C) Western blotting revealed that siRNA knockdown of HMOX-1 downregulated HMOX-1, Bcl-2 and Bcl-xl protein expression, but not Bax protein expression, in MDA-MB-231 and BT549 cells at $72 \mathrm{~h}$ post-transfection. Results are representative of three independent experiments. HMOX-1, heme oxygenase-1; C, control; PI, propidium iodide; Bcl-2, B cell lymphoma-2; Bcl-xl, B cell lymphoma-extra large; Bax, Bcl-2-like protein 4; FITC, fluorescein isothiocyanate. 

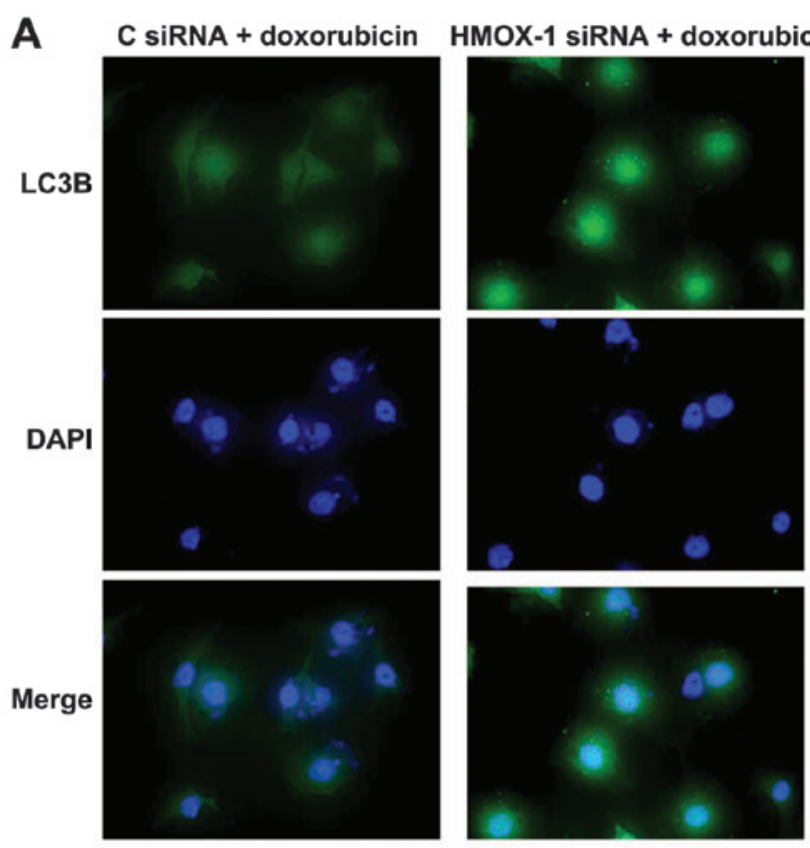

\section{C}

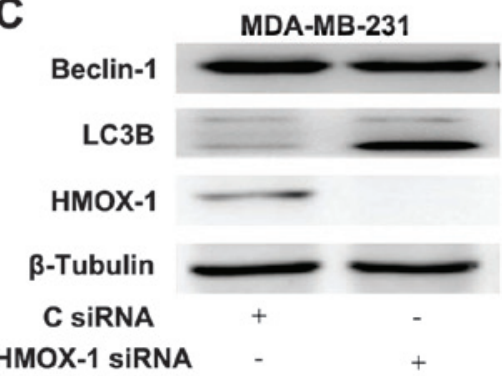

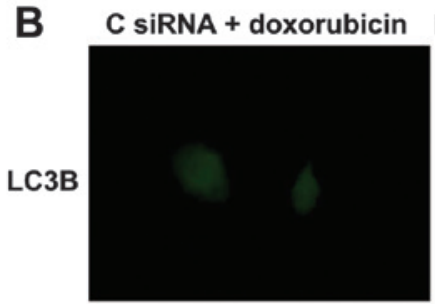

HMOX-1 SiRNA + doxorubicin
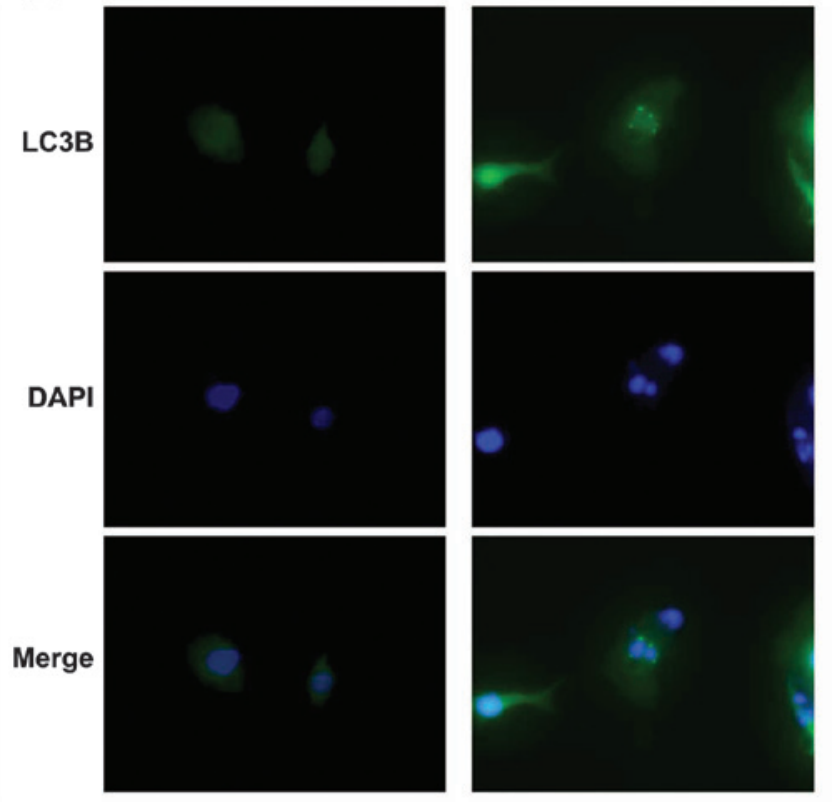

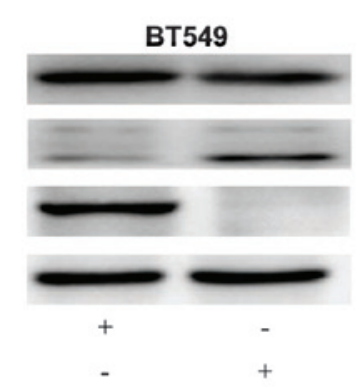

Figure 4. Knockdown of HMOX-1 enhances doxorubicin-induced autophagy in breast cancer cells. (A) MDA-MB-231 or (B) BT549 cells were transfected with C siRNA or $H M O X-1$ siRNA and then treated with $0.1 \mu \mathrm{g} / \mathrm{ml}$ doxorubicin for $48 \mathrm{~h}$. Immunofluorescent staining using a green fluorescent protein-tagged anti-LC3B antibody and fluorescence microscopy (magnification, x400) revealed that knockdown of $H M O X-1$ markedly increased the accumulation of autophagic vacuoles in the cancer cells. (C) Western blotting indicated that siRNA knockdown of $H M O X-1$ downregulated HMOX-1 and enhanced LC3B protein expression, but not Beclin-1 protein expression, in MDA-MB-231 and BT549 cells at $72 \mathrm{~h}$ post-transfection. Results are representative of three independent experiments. HMOX-1, heme oxygenase-1; LCB3, light chain 3B; C, control; siRNA, short interfering RNA.

HMOX-1 markedly increased the accumulation of autophagic vacuoles in doxorubicin-treated MDA-MB-231 and BT549 cells (Fig. 4A and B). Furthermore, western blotting confirmed that the expression of LC3B was upregulated in HMOX-1-silenced MDA-MB-231 and BT549 cells compared with that of the respective control cells, while Beclin-1 expression was unaffected (Fig. 4C).

\section{Discussion}

HMOX-1 is involved in the maintenance of cellular homeostasis and has a significant cytoprotective role (5). HMOX-1 is frequently upregulated in tumor tissues and promotes tumor growth and metastasis (8-14). Induction of HMOX-1 is often associated with chemoresistance in cancer cells, and the inhibition of HMOX-1 in combination with chemotherapy may enhance the efficacy of cancer treatment (24-26).

In the present study, it was demonstrated that HMOX-1 was overexpressed in breast cancer cell lines, and was also induced by doxorubicin. Therefore, the effect of HMOX-1 on the chemoresistance of breast cancer cells was investigated. Knockdown of HMOX-1 significantly enhanced the cytotoxicity of doxorubicin in MDA-MB-231 and BT549 cells. Therefore, the mechanism underlying the effects of knockdown of $H M O X-1$ on the cytotoxicity of doxorubicin was investigated. The Bcl-2 family includes pro-apoptotic (Bax, $\mathrm{Bcl}$-2-antagonist/killer 1 and $\mathrm{Bcl}$-2-associated death promoter) and anti-apoptotic (Bcl-2 and Bcl-xL) members and is a key regulator of apoptosis in cancer cells. Additionally, Bcl-2 and $\mathrm{Bcl}-\mathrm{xL}$ have been implicated in the resistance of cancer cells to chemotherapeutic drugs $(27,28)$. The results of the present study indicated that knockdown of $H M O X-1$ significantly enhanced doxorubicin-induced apoptosis and downregulated the expression of Bcl-2 and Bcl-xL in breast cancer cells. These findings indicated that HMOX-1 may modulate the survival of breast cancer cells via regulation of the expression of Bcl-2 and Bcl-xL.

Furthermore, knockdown of $H M O X-1$ enhanced doxorubicin-induced autophagy, without altering the expression of autophagic regulator Beclin-1 in MDA-MB-231 and BT549 cells. Autophagy is the process of lysosomal degradation, by which cells self-destruct, and has been associated with cell survival in various types of cancer (21). Autophagy is also able to protect cells from apoptosis and induce drug-resistance. 
Blocking autophagy restored anti-estrogen sensitivity to an MCF-7-derived anti-estrogen resistant cell line (29). Autophagy has also been implicated in the development of trastuzumab resistance in human epidermal growth factor receptor 2-positive breast cancer (30). A number of chemotherapeutic agents (anthracyclines and taxanes) are able to induce autophagy, and inhibition of autophagy increases drug toxicity via the induction of apoptosis in breast cancer cells $(31,32)$.

However, studies have indicated that excessive autophagy may result in autophagic cell death (also known as type II programmed cell death), which occurs in association with increased autophagosome formation in a caspase-independent manner (33). Scarlatti et al (34) reported that resveratrol, which is found in grapes and peanuts, induced Beclin-1-independent cytotoxic autophagy in MCF-7 cells. Di et al (35) demonstrated that breast cancer cells treated with doxorubicin and z-VAD-FMK, a general inhibitor of caspases, underwent cell death via a non-apoptotic pathway, characterized by dramatic accumulation of autophagic vacuoles.

Doxorubicin is one of the most commonly used chemotherapeutic drugs, and primarily functions by inhibiting topoisomerases and intercalating into the DNA double helix to interfere with DNA uncoiling, which induces cell death (36). Cell lines resistant to doxorubicin often exhibit defective apoptosis due to overexpression of Bcl-2 family proteins; therefore, chemotherapeutic agents that evoke autophagic cell death may be more cytotoxic in apoptosis-defective and drug-resistant tumor cells (37). In the present study, knockdown of HMOX-1 induced accumulation of autophagic vacuoles and markedly increased the expression of LC3B in breast cancer cells, without altering the expression of Beclin-1. Therefore, knockdown of $H M O X-1$ induced Beclin-1-independent autophagy in breast cancer cells. Under certain conditions, autophagy and apoptosis contribute to the rate of cell death as parallel pathways, and silencing Bcl-2 may induce autophagic cell death (31). Knockdown of $H M O X-1$ also reduced the expression of $\mathrm{Bcl}-2$ and promoted autophagy in breast cancer cells, suggesting that depletion of $H M O X-1$ may induce autophagic cell death by regulating the levels of $\mathrm{Bcl}-2$. These results demonstrate that knockdown of $H M O X-1$ enhanced the cytotoxic effects of doxorubicin and induced apoptosis and autophagy in breast cancer cells. These results indicate that HMOX-1 may have a role in tumor cell survival and chemoresistance in breast cancer. Additionally, HMOX-1 represents a potential therapeutic target, as targeting HMOX-1 may enhance the therapeutic efficacy of chemotherapy in breast cancer.

In conclusion, the present study demonstrated that HMOX-1 is overexpressed and able to be induced by doxorubicin in breast cancer cell lines. Knockdown of HMOX-1 enhanced the cytotoxicity of doxorubicin by downregulating anti-apoptotic genes and promoting apoptosis and autophagy in breast cancer cells. Therefore, HMOX-1 may represent a potential therapeutic target for breast cancer.

\section{Acknowledgements}

The present study was supported by the National Natural Science Foundation of China (no. 81172337) and the Guangdong Department of Science and Medicine Center (no. 2011A080300002).

\section{References}

1. Libson S and Lippman M: A review of clinical aspects of breast cancer. Int Rev Psychiatry 26: 4-15, 2014.

2. Peto R, Davies C, Godwin J, Gray R, Pan HC, Clarke M, Cutter D, Darby S, McGale P, Taylor C, et al; Early Breast Cancer Trialists' Collaborative Group (EBCTCG): Comparisons between different polychemotherapy regimens for early breast cancer: Meta-analyses of long-term outcome among 100,000 women in 123 randomised trials. Lancet 379: 432-444, 2012.

3. Videira M, Reis RL and Brito MA: Deconstructing breast cancer cell biology and the mechanisms of multidrug resistance. Biochim Biophys Acta 1846: 312-325, 2014.

4. Kontos CK, Christodoulou MI and Scorilas A: Apoptosis-related BCL2-family members: Key players in chemotherapy. Anticancer Agents Med Chem 14: 353-374, 2014.

5. Maines MD: The heme oxygenase system: A regulator of second messenger gases. Annu Rev Pharmacol Toxicol 37: 517-554, 1997.

6. Tenhunen R, Marver HS and Schmid R: The enzymatic conversion of heme to bilirubin by microsomal heme oxygenase. Proc Natl Acad Sci USA 61: 748-755, 1968.

7. Alam J, Shibahara S and Smith A: Transcriptional activation of the heme oxygenase gene by heme and cadmium in mouse hepatoma cells. J Biol Chem 264: 6371-6375, 1989.

8. Doi K, Akaike T, Fujii S, Tanaka S, Ikebe N, Beppu T, Shibahara S, Ogawa $M$ and Maeda H: Induction of haem oxygenase-1 nitric oxide and ischaemia in experimental solid tumours and implications for tumour growth. Br J Cancer 80: 1945-1954, 1999.

9. Maines MD and Abrahamsson PA: Expression of heme oxygenase-1 (HSP32) in human prostate: Normal, hyperplastic, and tumor tissue distribution. Urology 47: 727-733, 1996.

10. Torisu-Itakura H,Furue M,Kuwano M and Ono M: Co-expression of thymidine phosphorylase and heme oxygenase- 1 in macrophages in human malignant vertical growth melanomas. Jpn J Cancer Res 91: 906-910, 2000.

11. Hara E, Takahashi K, Tominaga T, Kumabe T, Kayama T, Suzuki H, Fujita H, Yoshimoto T, Shirato K and Shibahara S: Expression of heme oxygenase and inducible nitric oxide synthase mRNA in human brain tumors. Biochem Biophys Res Commun 224: 153-158, 1996.

12. Sunamura M, Duda DG, Ghattas MH, Lozonschi L, Motoi F, Yamauchi J, Matsuno S, Shibahara S and Abraham NG: Heme oxygenase-1 accelerates tumor angiogenesis of human pancreatic cancer. Angiogenesis 6: 15-24, 2003.

13. Cherrington JM, Strawn LM and Shawver LK: New paradigms for the treatment of cancer: The role of anti-angiogenesis agents. Adv Cancer Res 79: 1-38, 2000.

14. Was H, Cichon T, Smolarczyk R, Rudnicka D, Stopa M, Chevalier C, Leger JJ, Lackowska B, Grochot A, Bojkowska K, et al: Overexpression of heme oxygenase-1 in murine melanoma: Increased proliferation and viability of tumor cells, decreased survival of mice. Am J Pathol 169: 2181-2198, 2006.

15. Berberat PO, Dambrauskas Z, Gulbinas A, Giese T, Giese N, Künzli B, Autschbach F, Meuer S, Büchler MW and Friess H: Inhibition of heme oxygenase-1 increases responsiveness of pancreatic cancer cells to anticancer treatment. Clin Cancer Res 11: 3790-3798, 2005.

16. Noh SJ, Bae JS, Jamiyandorj U, Park HS, Kwon KS, Jung SH, Youn HJ, Lee H, Park BH, Chung MJ, et al: Expression of nerve growth factor and heme oxygenase-1 predict poor survival of breast carcinoma patients. BMC Cancer 13: 516, 2013.

17. Busserolles J, Megías J, Terencio MC and Alcaraz MJ: Heme oxygenase-1 inhibits apoptosis in Caco-2 cells via activation of Akt pathway. Int J Biochem Cell Biol 38: 1510-1517, 2006.

18. Chen GG, Liu ZM, Vlantis AC, Tse GM, Leung BC and van Hasselt CA: Heme oxygenase-1 protects against apoptosis induced by tumor necrosis factor-alpha and cycloheximide in papillary thyroid carcinoma cells. J Cell Biochem 92: 1246-1256, 2004.

19. Bolisetty S, Traylor AM, Kim J, Joseph R, Ricart K, Landar A and Agarwal A: Heme oxygenase-1 inhibits renal tubular macroautophagy in acute kidney injury. J Am Soc Nephrol 21: 1702-1712, 2010

20. Banerjee P, Basu A, Wegiel B, Otterbein LE, Mizumura K, Gasser M, Waaga-Gasser AM, Choi AM and Pal S: Heme oxygenase-1 promotes survival of renal cancer cells through modulation of apoptosis- and autophagy-regulating molecules. J Biol Chem 287: 32113-32123, 2012. 
21. Eisenberg-Lerner A, Bialik S, Simon HU and Kimchi A: Life and death partners: Apoptosis, autophagy and the cross-talk between them. Cell Death Differ 16: 966-975, 2009.

22. Shen HM and Codogno P: Autophagic cell death: Loch Ness monster or endangered species? Autophagy 7: 457-465, 2011.

23. Livak KJ and Schmittgen TD: Analysis of relative gene expression data using real time quantitative PCR and the 2(-Delta Delta C(T)) method. Methods 25: 402-408, 2001.

24. Mayerhofer M, Florian S, Krauth MT, Aichberger KJ, Bilban M, Marculescu R, Printz D, Fritsch G, Wagner O, Selzer E, et al: Identification of heme oxygenase-1 as a novel BCR/ABL-dependent survival factor in chronic myeloid leukemia. Cancer Res 64: 3148-3154, 2004.

25. Nuhn P, Künzli BM, Hennig R, Mitkus T, Ramanauskas T, Nobiling R, Meuer SC, Friess $\mathrm{H}$ and Berberat PO: Heme oxygenase- 1 and its metabolites affect pancreatic tumor growth in vivo. Mol Cancer 8: 37, 2009.

26. Al-Owais MM, Scragg JL, Dallas ML, Boycott HE, Warburton P, Chakrabarty A, Boyle JP and Peers C: Carbon monoxide mediates the anti-apoptotic effects of heme oxygenase- 1 in medulloblastoma DAOY cells via $\mathrm{K}^{+}$channel inhibition. J Biol Chem 287: 24754-24764, 2012.

27. Jäger R, Herzer U, Schenkel J and Weiher H: Overexpression of Bcl-2 inhibits alveolar cell apoptosis during involution and accelerates c-myc-induced tumorigenesis of the mammary gland in transgenic mice. Oncogene 15: 1787-1795, 1997.

28. Boise LH, González-García M, Postema CE, Ding L, Lindsten T, Turka LA, Mao X, Nuñez G and Thompson CB: Bcl-x, a bcl-2-related gene that functions as a dominant regulator of apoptotic cell death. Cell 74: 597-608, 1993.

29. Samaddar JS, Gaddy VT, Duplantier J, Thandavan SP, Shah M, Smith MJ, Browning D, Rawson J, Smith SB, Barrett JT, et al: A role for macroautophagy in protection against 4-hydroxytamoxifen-induced cell death and the development of antiestrogen resistance. Mol Cancer Ther 7: 2977-2987, 2008.
30. Cufí S, Vazquez-Martin A, Oliveras-Ferraros C, Corominas-Faja B, Cuyàs E, López-Bonet $\mathrm{E}$, Martin-Castillo $\mathrm{B}$, Joven $J$ and Menendez JA: The anti-malarial chloroquine overcomes primary resistance and restores sensitivity to trastuzumab in HER2-positive breast cancer. Sci Rep 3: 2469, 2013.

31. Sun WL, Chen J, Wang YP and Zheng H: Autophagy protects breast cancer cells from epirubicin-induced apoptosis and facilitates epirubicin-resistance development. Autophagy 7 : 1035-1044, 2011.

32. Veldhoen RA, Banman SL, Hemmerling DR, Odsen R, Simmen T, Simmonds AJ, Underhill DA and Goping IS: The chemotherapeutic agent paclitaxel inhibits autophagy through two distinct mechanisms that regulate apoptosis. Oncogene 32: 736-746, 2013

33. Denton D, Nicolson S and Kumar S: Cell death by autophagy: Facts and apparent artefacts. Cell Death Differ 19: 87-95, 2012.

34. Scarlatti F, Maffei R, Beau I, Codogno P and Ghidoni R: Role of non-canonical Beclin 1-independent autophagy in cell death induced by resveratrol in human breast cancer cells. Cell Death Differ 15: 1318-1329, 2008.

35. Di X, Shiu RP, Newsham IF and Gewirtz DA: Apoptosis, autophagy, accelerated senescence and reactive oxygen in the response of human breast tumor cells to adriamycin. Biochem Pharmacol 77: 1139-1150, 2009.

36. Box VG: The intercalation of DNA double helices with doxorubicin and nogalamycin. J Mol Graph Model 26: 14-19, 2007.

37. Wirawan E, Vande Walle L, Kersse K, Cornelis S, Claerhout S, Vanoverberghe I, Roelandt R, De Rycke R, Verspurten J, Declercq W, et al: Caspase-mediated cleavage of Beclin-1 inactivates Beclin-1-induced autophagy and enhances apoptosis by promoting the release of proapoptotic factors from mitochondria. Cell Death Dis 1: e18, 2010. 